\title{
LA ESCUELA AUSTRIACA Y EL VALUE INVESTING: UNA APROXIMACIÓN (I)
}

\author{
The Austrian School and Value Investing: \\ An Approximation (I)
}

\author{
PABLO YUSTA*
}

Fecha de recepción: 20 de noviembre de 2020

Fecha de aceptación: 14 de abril de 2021

Resumen: No es necesario conocer la Escuela Austriaca para hacer Value Investing, ni es necesario conocer el Value Investing para saber de Escuela Austriaca; pero es interesante detectar las ventajas cruzadas que aparecen al abordar ambos campos de estudio. Este primer artículo introductorio de la trilogía quedará dividido en tres partes: (1) definición de los dos conceptos y comparación con sus análogos mainstream, (2) resumen de las similitudes y compatibilidades entre ambos conceptos, (3) análisis de las discrepancias e incompatibilidades puestas de manifiesto en el debate académico actual sobre su nivel de coincidencia.

Palabras clave: Escuela Austriaca, Value Investing.

Clasificación JEL: B13, B25, B53, E14, G11, G32.

Abstract: There is no need to know the Austrian School to practice Value Investing nor to know what Value Investing is to understand the Austrian School; but it is interesting to detect the cross advantages that appear when approaching both fields of study. This first introductory article of the trilogy will be divided into three parts: (1) definition of the two concepts and comparison with their mainstream analogues, (2) summary of the points in common between both concepts, (3) analysis of the incompatibilities revealed in the current academic debate on this topic.

\footnotetext{
* Pablo Yusta (p.yusta.0112@gmail.com) es Máster en Economía de la Escuela Austriaca y doctorando de la Universidad Rey Juan Carlos de Madrid
} 
Keywords: Austrian School, Value Investing.

JEL classification: B13, B25, B53, E14, G11, G32.

\section{PREÁMBULO}

Este artículo pertenece a una colección de tres artículos titulados: la Escuela Austriaca y el Value Investing: una Aproximación, que pretende hacer un repaso de la bibliografía a la que he tenido acceso hasta la fecha sobre estos dos enfoques. La distribución de las publicaciones será:

- I: en el primer artículo se explicará el terreno de juego donde vamos a adentrarnos, definiremos y discutiremos los conceptos.

- $\quad$ II: en el segundo artículo se analizarán los beneficios que conlleva conocer la Escuela Austriaca a la hora de invertir.

- III: en el tercer artículo se analizarán los beneficios que conlleva adquirir experiencia en el análisis de inversiones a la hora de comprender la teoría económica austriaca.

El fin divulgador de este trabajo pretende llegar al máximo número de personas, de diferentes ámbitos y niveles de especialización. Los conceptos se expondrán de manera resumida y concisa, aunque no por eso se perderá solidez o rigor sobre los temas tratados.

Estuve reflexionando sobre cómo escribir de una manera sencilla, pero que a la vez resultara de utilidad a personas especializadas, y llegué a la conclusión de que debía utilizar cuadros conceptuales. Son una herramienta útil que sirve tanto a las personas menos especializadas porque pueden formar un esquema (estantería) mental que irán llenando de contenido con futuras lecturas de mayor profundidad; como a las personas más especializadas ya que les ayudará a clasificar y a tener ordenada toda la información de la que ya disponen. No se sorprendan si encuentras varios a lo largo de los artículos. 
Algunos seguidores de la Escuela Austriaca practican el Value Investing ${ }^{1} \mathrm{y}$, a su vez, algunos value investors se declaran seguidores de la Escuela Austriaca. ${ }^{2}$ Es evidente que existe cierta cercanía y consonancia entre ambos enfoques, pero en ningún caso debemos de pensar que uno es condición sine qua non para la existencia del otro: ni un inversor en valor deja de serlo por no leer a Mises, ni un economista austriaco deja de serlo por invertir en fondos índice. Es más, Warren Buffett, el mejor value investor de todos los tiempos, ha mencionado en más de una ocasión que si se lee el capítulo 12 de La Teoría General de la Ocupación, el Interés y el Dinero, de John Maynard Keynes, uno no tiene que leer nada más y puede apagar la televisión 3 . Además, en palabras de Buffett en 1989: “Reading Keynes will make you smarter about securities and markets, I'm not sure reading most economists would do the same".

Sin embargo, en este capítulo vamos a demostrar que se puede considerar que la Escuela Austriaca de Economía y el método de Inversión en Valor son compatibles porque tienen una visión similar del mundo, y son complementarias porque lo enfocan de manera diferente. Quijano Durán (2011) comenta a este respecto:

${ }^{1}$ Utilizaremos indistintamente las expresiones Inversión en Valor y Value Investing. Sin embargo, los conceptos son matizables ya que se tiende a relacionar al término en español con la inversión en activos extremadamente baratos (bajo PER, bajo EV/EBITDA y bajo precio sobre valor en libros), y a la expresión inglesa con un concepto más amplio que incluye cualquier tipo de inversión en activos que valgan más de lo que se está pagando. Cuando utilice cualquiera de las dos expresiones, me referiré a esta segunda acepción más general.

2 Como ejemplo de economistas que consideren que existe esta consonancia: Juan Ramón Rallo, Diego E. Quijano Durán, Chris Leithner, Juan Ignacio del Castillo; y de value investors que hayan encontrado en la Escuela Austriaco una teoría en la que apoyarse: Francisco García Paramés, Iván Martín, Álvaro Guzmán de Lázaro, Emérito Quintana, Juan Diego Huerta de Soto.

${ }^{3}$ En una entrevista a Business Wire en Noviembre de 2011, Buffett dijo: «If you understand chapter 8 and 20 of The Intelligent Investor (Benjamin Graham, 1949) and chapter 12 of The General Theory (John Maynard Keynes, 1936), you don't need to read anything else and you can turn off your TV». 
«Son compatibles porque ambas consideran que no se puede predecir en el mundo económico, y que lo más que se puede hacer es identificar los patrones y características que tienden a provocar los resultados buscados. Son complementarias porque el Value Investing tiene como objetivo final generar ganancias al inversor ayudándolo a escoger las empresas en donde invertir, mientras que la Escuela Austriaca trata de comprender los fenómenos económicos y los procesos dentro de los cuales operan las empresas». ${ }^{4}$

Además, la influencia entre ambas metodologías resulta beneficiosa para ambos profesionales. «Para el economista, conocer el método de la inversión en valor le permite profundizar el conocimiento de la función empresarial y un método práctico, que ha sido muy exitoso, de la realización del cálculo económico para determinar el mejor uso del capital [...]. Al inversor le ofrece un marco teórico para comprender mejor el papel del empresario y los fenómenos económicos (ciclos) y detectar temprano patrones peligrosos y así protegerse». ${ }^{5}$

Según estas consideraciones, y repito, sin considerar que una es condición necesaria para que exista la otra, el inversor que se base en la teoría de la Escuela Austriaca, tendrá una especie de caja de herramientas ${ }^{6}$ para identificar patrones de tendencia peligrosos y poder reducir la incertidumbre inerradicable mientras opera en el mercado. En este contexto, podemos entender las citas de Grant (1996) y de Leithner (2005):

«If you know that the economy is dominated by the time-bound structures of production and speculation, the world comes into clearer focus. [...] What we do is look for extremes in markets: very undervalued or very overvalued. Austrian theory has certainly given us an edge. When you have a theory to work from, you avoid the problem that comes with stumbling around in the dark over chairs and nightstands. At least you can begin to visualize in the dark, which is where we all work. The future is always unlit. But

\footnotetext{
4 Quijano Durán (2011). Cursivas añadidas por el autor.

5 Quijano Durán (2012), Cursivas añadidas por el autor.

6 Lo que Emérito Quintana (2016) denomina «caja de herramientas conceptual».
} 
with a body of theory, you can anticipate where the structures might lie».7 (Grant, 1996).

«To value investors, Austrian economics is (or should be) compelling because it subsumes real economic and financial events within justifiable laws of human action. Unlike mainstream economic and finance, Austrians not only acknowledge but also emphasise the importance of entrepreneurship [...]. In turn, value investing, and the results achieved by prominent value investors may interest Austrians because Grahamites hold [...] views that are compatible to Austrian views with respect to important economic and financial principles. Value investors also illustrate the positive results that entrepreneurial action can achieve. Despite the many follies of governments and mainstream economists and investors, which often prompt value investors and Austrians alike to adopt rather dour short-term outlooks, both Grahamites and Misesians are long term optimists. To adopt their approaches to economics and investment is ultimately to affirm a basic faith in human nature, capitalism, one's country and a future that will be at least as prosperous as the present». ${ }^{8}$ (Leithner, 2005).

\section{LA ESCUELA AUSTRIACA}

«Man himself is the beginning and the end of every economy».

(Carl Menger, 1892).

En esta sección, presentaremos una breve descripción de la Escuela Austriaca de Economía. ${ }^{9}$ Por supuesto, debido a su amplitud de

7 Encontramos la cita original en la entrevista realizada a James Grant titulada: "The trouble with Prosperity", publicada en 1996 en la Austrian Economics Newsletter, Vol. 16, No 4 .

8 Encontramos la cita original en el artículo de Chris Leithner titulado: "Ludwig von Mises, Meet Benjamin Graham: Value Investing from an Austrian Point of View", preparado para la conferencia Austrian Economics and Financial Markets en 2005 en Las Vegas.

${ }^{9}$ El origen de la Escuela Austriaca lo podemos situar en 1871 con la publicación del libro Principios de Economía Política de Carl Menger y continúa desarrollándose hasta el día de hoy. Para los que se inicien en este campo, vamos a hacer un repaso de 
campos de estudio, solo haremos un repaso superficial. Describir la Escuela Austriaca en pocas palabras es una tarea difícil. Para que el lector pueda seguir el hilo lógico durante la exposición, comenzaré con una breve introducción y, posteriormente, he optado por presentar de una manera hilada los tres niveles de análisis que existen en esta escuela de pensamiento económico: la Acción Humana, las Relaciones de Intercambio y las Instituciones Sociales Evolutivas; incluidos en Carl Menger (1871).

Si el lector desea iniciarse en el tema, recomiendo la lectura del libro del profesor Jesús Huerta de Soto (2012) titulado La Escuela Austriaca: Mercado y creatividad empresarial y para los que deseen especializarse o conocer un poco más, no tendrán más que recurrir a la cuidada bibliografía seleccionada al final de ese mismo libro, en el que encontrarán marcadas con un asterisco las obras más recomendadas sobre esta escuela de pensamiento. En su discurso pronunciado al recibir el doctorado honoris causa en Ciencias Sociales por la Universidad Francisco Marroquín en 2009, Huerta de Soto realizó una precisa descripción del pensamiento austriaco:

«La Escuela Austriaca es una escuela muy humanista y multidisciplinar, que desarrolla sus aportaciones teóricas partiendo del ser humano; es decir, de los hombres y mujeres tal y como son en realidad, y por tanto no centrándose en un estereotipo, especie de ser robotizado, ese homo economicus maximizador de beneficios, que es el protagonista de los modelos matemáticos de las diferentes versiones de la Escuela Neoclásica (desde los neokeynesianos a los teóricos de Chicago).

Según los austriacos, el protagonista de todos los fenómenos sociales es el empresario, entendido como el ser humano dotado de

algunos de los autores más importantes de orden de más antiguo a más reciente: Carl Menger, Eugen Böhm-Bawerk, Ludwig von Mises, Friedrich Hayek (premio Nobel 1974), Murray Rothbard, Israel Kirzner, Peter Boetke o Jesús Huerta de Soto. Además, muchos otros economistas han admitido que se han basado en los paradigmas austriacos para desarrollar sus ideas, entre ellos destacan: James Buchanan (1986), Ronald Coase (1991), Douglass North (1993), Vernon Smith (2002), Oliver Williamson (2009), Elinor Ostrom (2009). Entre paréntesis, el año en el que recibieron el premio Nobel. La anterior enumeración ha sido confeccionada de acuerdo con Juan Ramón Rallo (2019). 
una innata capacidad, a la vez creativa y coordinadora, para descubrir los fines que le merecen la pena y actuar para alcanzarlos». ${ }^{10}$

Por lo tanto, la Escuela Austriaca de economía, partiendo del individualismo metodológico ${ }^{11}$, se centra en estudiar las acciones humanas, es decir, las acciones de las personas de carne y hueso. Esto del individualismo no quiere decir que se considere que no existen o que no sean relevantes las asociaciones o las agrupaciones en la sociedad, sino que, para estudiarlas, no se pueden percibir como un agregado con personalidad propia. Sobre esas valoraciones subjetivas que llevan a las personas a actuar, la Escuela Austriaca no entra en analizar si son más o menos correctas, se limita a «reconocer el hecho incontrovertible de que cuando las personas actúan lo hacen en pos de los fines que más valoran subjetivamente en ese momento y utilizan los medios a su disposición que consideran más útiles para alcanzarlos». ${ }^{12}$

Esas personas al tratar de alcanzar los fines que son de su propio interés y haciendo uso de la información de la que disponen, se coordinan con el resto para intercambiar aquellos bienes y servicios que valoran menos y obtener los que valoran más. Aquí es donde nace la economía, en el estudio de los mercados donde las personas realizan voluntariamente intercambios de bienes y servicios para alcanzar sus respectivos fines personales. En un intercambio, los actores participantes salen beneficiados porque obtienen lo que deseaban, dando a cambio algo que valoran menos (si no, no realizarían el intercambio). En este aspecto, «el énfasis de la escuela austriaca [...] está en el estudio del mercado como un proceso de interrelaciones entre personas, cada una de las cuales está persiguiendo sus fines y va adaptando y modificando su comportamiento (y los propios fines) conforme va actuando ${ }^{13}$ de acuerdo con las circunstancias que encuentra en el mundo externo y la

10 Huerta de Soto (2009).

11 El individualismo metodológico sostiene que todos los fenómenos sociales son retrotraibles a la acción humana individual y que, por tanto, deben ser estudiados a partir de los seres humanos, los individuos.

12 Quijano Durán (2012), p.259. Cursivas añadidas por el autor.

13 Para profundizar sobre el concepto de serendipidad ver Huerta de Soto (2015) nota pág. 47, y Artur Ceolin (2019): “Contrastando el papel de la serendipidad en las teorías austriaca y neoclásica". 
capacidad de alcanzarlos». ${ }^{14}$ Esta es la razón de que la manera de percibir y entender los mercados sea «como un proceso competitivo, que jamás se encuentra en equilibrio y que no puede ser diseñado ni controlado centralizadamente por nadie». ${ }^{15}$

Sin embargo, aunque parezca que existe una insalvable falta de regularidad porque el ser humano no actúa de manera robotizada, en esa realidad dinámica descrita, surgen órdenes espontáneos que permiten una mejor coordinación de los planes y acciones de los individuos en la sociedad. Así, Martínez Meseguer (2006) afirma que un orden espontáneo aparece:

«[...] fruto de una serie de regularidades (hábitos, costumbres), surgidas de los intentos de adaptación de los individuos que integran la sociedad a las circunstancias que les afectan en cada momento y lugar, y que posibilitan la realización de proyecciones de futuro extraídas de dicha ordenación. Después de dilatados procesos de prueba y error se van alcanzando soluciones cada vez más adecuadas a los problemas. Dichas soluciones que demuestran ser las más apropiadas se aprenden y se transmiten a futuras generaciones por los individuos y grupos que las adoptan..»16

Por tanto, los órdenes sociales espontáneos surgen por medio de procesos evolutivos de prueba y error $\mathrm{y}$, gracias a un entorno de respeto a la propiedad privada (y a su transmisión), van formalizándose, naciendo así las instituciones sociales. ${ }^{17}$ Las sociedades más avanzadas serán precisamente aquellas que contengan una

14 Quijano Durán (2012), p.260. Cursivas añadidas por el autor.

15 Huerta de Soto (2009).

16 Martínez Meseguer, C. (2006) p.195-196.

17 Según Quintana (2016) las instituciones se pueden definir como: «esquemas pautados de comportamiento que surgen espontánea y evolutivamente gracias a los intentos de adaptación de los individuos a las condiciones sociales de cada momento en la búsqueda de sus propios beneficios. Estas conductas son imitadas por la mayoría de individuos si facilitan la consecución del mayor número posible de objetivos individuales. Esta imitación conlleva a su vez un proceso de ensayo y error en el cual preponderan los grupos de individuos que eligen el comportamiento más útil para resolver sus problemas y lograr sus objetivos, De tal forma que se convierten en hábitos y costumbres que van modificándose con el tiempo, y que aprovechan una gran cantidad de conocimiento tácito acumulado durante siglos. Podemos nombrar, por ejemplo: el lenguaje, el derecho, la familia, la moral, o el dinero». 
serie de instituciones sociales como las normas jurídicas, el mercado y el dinero, ya que posibilitan un aumento exponencial en los intercambios, la división del conocimiento y la reducción de la incertidumbre.

En resumen, el mercado y las demás instituciones sociales son fruto de la acción humana, pero no del diseño humano. Así, los diferentes intercambios que se producen a gran escala en el mercado son consecuencia directa de las personas buscando su propio interés y haciendo uso de la información de la que dispone. Por tanto, esta cooperación que se produce a través de los intercambios, no es fruto del diseño centralizado de ninguna mente humana, sino de las acciones humana de millones de individuos durante largos periodos dilatados de tiempo. Nadie ha decidido de manera deliberada la estructura del mercado, al igual que sucede con otras instituciones sociales como el lenguaje, la cultura, las costumbres... Es decir, y aunque suene contraintuitivo, el mercado es una consecuencia no intencionada de nuestras acciones.

«Aunque pudiera parecer que existe una mano invisible que controla a todas las personas para lograr una exitosa operación a gran escala tal mano invisible no existe: alcanzamos un orden cooperativo de manera espontánea. Por un lado, respetando la libertad y la propiedad ajena y por otro, ejecutando de manera descentralizada planes empresariales en régimen de competencia que aprovechan la información dispersa que nos proporciona el sistema de precios. Eso, y no otra cosa, es el libre mercado». ${ }^{18}$

Antes de entrar a explicar los principios básicos de la filosofía del Value Investing, considero que puede aportar valor exponer el cuadro comparativo ${ }^{19}$ entre los Principios esenciales de la Escuela Austriaca y la Neoclásica. Así, el lector podrá comprobar de manera sencilla los postulados que defiende este paradigma y compararlos con los de la corriente dominante en economía.

18 Juan Ramón Rallo (2019): La Ruta Value.

19 El cuadro es un extracto del que aparece en el primer capítulo de Huerta de Soto (2012). 


\section{CUADRO 1: PARADIGMA AUSTRIACO VS. PARADIGMA NEOCLÁSICO}

\begin{tabular}{|c|c|}
\hline PARADIGMA AUSTRIACO & PARADIGMA NEOCLÁSICO \\
\hline $\begin{array}{l}\text {-Concepto de lo económico (principio } \\
\text { esencial)- }\end{array}$ & $\begin{array}{l}\text {-Concepto de lo económico (principio } \\
\text { esencial)- }\end{array}$ \\
\hline $\begin{array}{l}\text { Teoría de la acción humana entendida } \\
\text { como un proceso dinámico } \\
\text { (praxeología). }\end{array}$ & $\begin{array}{l}\text { Teoría de la decisión: maximización } \\
\text { sometida a restricciones (concepto } \\
\text { estrecho de racionalidad). }\end{array}$ \\
\hline -Punto de vista metodológico- & -Punto de vista metodológico- \\
\hline Subjetivismo. & $\begin{array}{l}\text { Estereotipo del individualismo } \\
\text { metodológico (objetivista). }\end{array}$ \\
\hline -Protagonista de los procesos sociales- & -Protagonista de los procesos sociales- \\
\hline Empresario creativo. & Homo economicus. \\
\hline $\begin{array}{l}\text {-Posibilidad de que los actores se } \\
\text { equivoquen a priori y naturaleza del } \\
\text { beneficio empresarial- }\end{array}$ & $\begin{array}{l}\text {-Posibilidad de que los actores se } \\
\text { equivoquen a priori y naturaleza del } \\
\text { beneficio empresarial- }\end{array}$ \\
\hline $\begin{array}{l}\text { Se concibe la posibilidad de cometer } \\
\text { errores empresariales puros que } \\
\text { hubieran podido evitarse con más } \\
\text { perspicacia empresarial para darse } \\
\text { cuenta de las oportunidades de } \\
\text { ganancia. }\end{array}$ & $\begin{array}{c}\text { No se concibe que existan errores de los } \\
\text { que uno pueda arrepentirse, todas las } \\
\text { decisiones pasadas se racionalizan en } \\
\text { términos de costes y beneficios. Los } \\
\text { beneficios empresariales se consideran } \\
\text { como la renta de un factor más } \\
\text { productivo. }\end{array}$ \\
\hline -Concepción de la información- & -Concepción de la información- \\
\hline $\begin{array}{c}\text { El conocimiento y la información son } \\
\text { subjetivos, están dispersos y cambian } \\
\text { constantemente (creatividad } \\
\text { empresarial). Distinción radical entre } \\
\text { conocimiento científico (objetivo) y } \\
\text { práctico (subjetivo). }\end{array}$ & $\begin{array}{l}\text { Se supone información plena (en } \\
\text { términos ciertos o probabilísticos) de } \\
\text { fines y medios que es objetiva y } \\
\text { constante. No distinguen entre } \\
\text { conocimiento práctico (empresarial) y } \\
\text { científico. }\end{array}$ \\
\hline -Foco de referencia- & -Foco de referencia- \\
\hline $\begin{array}{l}\text { Proceso general con tendencia } \\
\text { coordinadora. No se distingue la micro } \\
\text { y la macro: todos los problemas } \\
\text { económicos se estudian de forma } \\
\text { interrelacionada. }\end{array}$ & $\begin{array}{c}\text { Modelo de equilibrio (general o parcial). } \\
\text { Separación entre la micro y la } \\
\text { macroeconomía. }\end{array}$ \\
\hline -Concepto de competencia- & -Concepto de competencia- \\
\hline Proceso de rivalidad empresarial. & $\begin{array}{l}\text { Situación o modelo de "competencia } \\
\text { perfecta". }\end{array}$ \\
\hline
\end{tabular}




\begin{tabular}{|c|c|}
\hline PARADIGMA AUSTRIACO & PARADIGMA NEOCLÁSICO \\
\hline -Concepto de coste- & -Concepto de coste- \\
\hline $\begin{array}{l}\text { Subjetivo (depende de la perspicacia } \\
\text { empresarial para descubrir nuevos } \\
\text { fines alternativos). }\end{array}$ & $\begin{array}{l}\text { Objetivo y constante (se puede } \\
\text { reconocer por un tercero y medir). }\end{array}$ \\
\hline -Formalismo- & -Formalismo- \\
\hline $\begin{array}{l}\text { Lógica verbal (abstracta y formal) que } \\
\text { da entrada al tiempo subjetivo y a la } \\
\text { creatividad humana. }\end{array}$ & $\begin{array}{l}\text { Formalismo matemático (lenguaje } \\
\text { simbólico propio del análisis de } \\
\text { fenómenos atemporales y constantes). }\end{array}$ \\
\hline -Relación con el mundo empírico- & -Relación con el mundo empírico- \\
\hline $\begin{array}{l}\text { Razonamientos apriorístico-deductivos: } \\
\text { separación radical y, a la vez, } \\
\text { coordinación entre teoría (ciencia) e } \\
\text { historia (arte). La historia no puede } \\
\text { contrastar teorías. }\end{array}$ & $\begin{array}{l}\text { Contrastación empírica de las hipótesis } \\
\text { (al menos retóricamente). }\end{array}$ \\
\hline -Posibilidades de predicción específica- & -Posibilidades de predicción específica- \\
\hline $\begin{array}{l}\text { Imposible, pues lo que suceda depende } \\
\text { de un conocimiento empresarial futuro } \\
\text { aún no creado. Sólo son posibles pattern } \\
\text { predictions de tipo cualitativo y teórico } \\
\text { sobre las consecuencias de } \\
\text { descoordinación del intervencionismo. }\end{array}$ & $\begin{array}{l}\text { La predicción es un objetivo que se } \\
\text { busca de forma deliberada. }\end{array}$ \\
\hline -Responsable de la predicción- & -Responsable de la predicción- \\
\hline El empresario. & El analista económico (ingeniero social). \\
\hline -Estado actual del paradigma- & -Estado actual del paradigma- \\
\hline $\begin{array}{c}\text { Notable resurgimiento en los últimos } 25 \\
\text { años (especialmente tras la crisis del } \\
\text { keynesianismo y la caída del } \\
\text { socialismo). }\end{array}$ & Situación de crisis y cambio acelerado. \\
\hline $\begin{array}{l}\text {-Cantidad de "capital humano" } \\
\text { invertido- }\end{array}$ & $\begin{array}{l}\text {-Cantidad de "capital humano" } \\
\text { invertido- }\end{array}$ \\
\hline Minoritario, pero creciente. & $\begin{array}{l}\text { Mayoritario y con signos de } \\
\text { dispersamiento y disgregación. }\end{array}$ \\
\hline -Tipo de "capital humano" invertido- & -Tipo de "capital humano" invertido- \\
\hline $\begin{array}{l}\text { Teóricos y filósofos multidisciplinares. } \\
\text { Liberales radicales. }\end{array}$ & $\begin{array}{l}\text { Especialistas en intervenciones } \\
\text { económicas (piecemeal social } \\
\text { engineering). Grado muy variable de } \\
\text { compromiso con la libertad. }\end{array}$ \\
\hline
\end{tabular}




\section{EL VALUE INVESTING}

«All intelligent investing is value investing -acquiring more than you are paying for-. You must value the business in order to value the stock».

(Charlie Munger, 2005)

El Value Investing es un estilo de inversión que ha pasado a ser una filosofía de inversión, pero que no ha llegado a la categoría de ciencia de la inversión. ${ }^{20}$ Se ha convertido en filosofía de inversión ya que «tras décadas de aprendizaje práctico y empresarial sobre los mercados financieros y a través de un método de ensayo, error, imitación y generación de ideas, han ido intuyendo y acercándose asintóticamente a los principios que rigen su disciplina, pero no han terminado de dar con la respuesta ${ }^{21}$. Esto ha sido posible, entre otras cosas, porque en el mundo del Value Investing destaca la profunda generosidad de los inversores más prestigiosos, ya que, la mayoría de ellos, ha plasmado en algún libro o revista los puntos clave de su método de inversión. Convertirse en una ciencia de la inversión es un cambio significativo, pasar de ser un conjunto de anécdotas y experiencias basadas en hechos empíricos a una teoría sólida, significaría que tendríamos a nuestra disposición las gafas teóricas para poder observar la realidad de manera más clara y transparente, sea cual sea esta. ${ }^{22}$ Sobre la posibilidad

${ }^{20} \mathrm{El}$ origen del value investing lo podemos situar en Benjamin Graham, profesor de la Universidad de Columbia, con la publicación de sus dos libros más importantes: Security Analysis en 1934 y El Inversor Inteligente en 1949. Es cierto que el término value investing no se le puede atribuir a este autor, ya que Graham hablaba de intelligent investing y no de value investing. Sobre la diferencia entre estos dos conceptos, recomiendo leer el artículo de Andrei Trucmel publicado en 2019 titulado: "Malinterpretando el value investing". Para los que se inicien en este campo, vamos a hacer un repaso de algunos de los inversores, que, de manera más o menos directa, están relacionados con esta filosofía de inversión: Benjamin Graham, Warren Buffett, Charlie Munger, Walter Schloss, Shelby Davis, George Soros, Peter Lynch, Jim Rogers, Joel Greenblatt, Sir John Templeton, Philip Fisher, Seth Klarman, Bill Ackman, Christopher H. Browne o Francisco García Paramés. La enumeración se ha hecho de acuerdo con Buffett (1984) y he añadido en cursiva algunos otros.

21 Emérito Quintana (2016).

22 En otras palabras, dejaríamos de ser el extraterrestre Gurb en la novela de Eduardo Mendoza (1991) titulada Sin Noticas de Gurb, que sólo es capaz de entender la 
de convertirse en una ciencia de la inversión, Juan Ramón Rallo (2010) dice al respecto:

«Por otro lado, los value investors deberán de estar dispuestos a dejar de ser una "filosofía de inversión" para convertirse en una "ciencia de la inversión", con todo lo que ello implica: rigor en las categorías [...], preocupación porque sus proposiciones sean consistentes entre sí y con el resto de leyes económicas, mayor esfuerzo por articular un conocimiento difícilmente articulable y, como consecuencia de lo anterior, un progresivo abandono de su anecdotario de experiencias personales y de estudios de caso en pos de una mayor modelización de las técnicas inversoras hasta donde sea técnicamente posible.

En definitiva, los value investors deberán aceptar "descargar" un conocimiento que hasta hoy se ha desarrollado esencialmente en torno a intuiciones y comprensiones personales del mercado, y para ello deberán formalizarlo y someterlo a los estándares del razonamiento científico. $»^{23}$

Al igual que ocurría con la Escuela Austriaca, presentar los principios del value investing en unas pocas líneas es complicado, por lo que voy a exponer los tres principios básicos: margen de seguridad, la parábola del Sr. Mercado y la perspectiva empresarial del inversor; que fueron recogidos en la que es considerada la biblia del value investing 24 : El Inversor Inteligente de Benjamin Graham (1949).

Podemos identificar el value investing como «una metodología que enfatiza el análisis de los fundamentos financieros de las empresas. El enfoque no está en el comportamiento histórico del precio de la acción en el mercado de valores, sino en intentar

realidad como unidades de carbono que se mueven, emiten frecuencias, intercambian bienes, etc.

23 Juan Ramón Rallo (2010).

24 Para Warren Buffett este libro es «by far the best book on investing ever written». Además, añade: "Chapters 8 and 20 have been the bedrock of my investing activities for more than 60 years / I suggest that all investors read those chapters and reread them every time the market has been especially strong or weak." 
determinar cuál es el valor razonable o rango de valores razonables ${ }^{25}$ de la empresa basándose en su historial financiero, una evaluación de sus características, su sector, su administración y sus ventajas competitivas». ${ }^{26}$ De esta manera, el éxito de la inversión se basa en comprar activos a un precio de mercado inferior a nuestra estimación subjetiva de valor razonable. La diferencia entre precio y valor se denomina margen de seguridad, y es considerado el concepto más importante de esta filosofía de inversión:

«Confronted with a challenge to distil the secret of sound investment into three words, we venture the motto, Margin of Safety». ${ }^{27}$

Cuanto mayor sea el margen de seguridad menores serán las pérdidas potenciales y mayores las posibilidades de obtener un beneficio económico. Como nuestras valoraciones son subjetivas y no controlamos el precio de cotización de los activos, tiene sentido que tratemos de reducir la incertidumbre en la inversión por medio de amplios márgenes de seguridad ${ }^{28}$, seleccionando empresas con ventajas competitivas ${ }^{29}$ y de calidad ${ }^{30}$.

En segundo lugar, si el margen de seguridad es el pilar fundamental de la inversión en valor, no menos afamada es la parábola del Sr. Mercado en la que Graham (1949) describe al mercado como un péndulo que oscila constantemente entre un optimismo insostenible (que hace que las acciones sean demasiado caras) y un pesimismo injustificado (que hace que sean demasiado baratas). El inversor inteligente será, por tanto, capaz de entender el concepto

${ }^{25}$ Aunque se discutirá más adelante en este capítulo, es importante entender que la valoración de activos es siempre subjetiva, por lo tanto, este valor razonable o rango de valores razonables dependerán de las estimaciones individuales de cada inversor.

${ }^{26}$ Quijano Durán (2012), p.262. Cursivas añadidas por el autor.

27 Benjamin Graham en The Intelligent Investor (1949).

28 Sobre el concepto margen de seguridad, ver el libro de Seth Klarman (1991) titulado Margin of Safety.

${ }^{29}$ Sobre el concepto de ventajas competitivas, ver el libro de Pat Dorsey (2008) titulado The Little Book That Builds Wealth.

30 El concepto de empresas de calidad está muy ligado al anterior de ventajas competitivas, recomiendo leer el libro de L. Cunningham, T. Eide y P. Hargreaves (2016) titulado Quality Investing. 
y, así, vender a optimistas y comprar a pesimistas. Warren Buffett (1987) explica al respecto:

«Ben Graham, my friend and teacher, long ago described the mental attitude toward market fluctuations that I believe to be most conducive to investment success. He said that you should imagine market quotations as coming from a remarkably accommodating fellow named Mr. Market who is your partner in a private business. Without fail, Mr. Market appears daily and names a price at which he will either buy your interest or sell you his.

Even though the business that the two of you own may have economic characteristics that are stable, Mr. Market's quotations will be anything but. For, sad to say, the poor fellow has incurable emotional problems. At times he feels euphoric and can see only the favorable factors affecting the business. When in that mood, he names a very high buy-sell price because he fears that you will snap up his interest and rob him of imminent gains. At other times he is depressed and can see nothing but trouble ahead for both the business and the world. On these occasions, he will name a very low price, since he is terrified that you will unload your interest on him.

Mr. Market has another endearing characteristic: He doesn't mind being ignored. If his quotation is uninteresting to you today, he will be back with a new one tomorrow. Transactions are strictly at your option. Under these conditions, the more manic-depressive his behavior, the better for you».

En tercer lugar, el Value Investing consiste en invertir desde un punto de vista empresarial, es decir, debe relacionarse con nuestra perspicacia empresarial a la hora de valorar correctamente los activos que estemos analizando. Bejamin Graham (1949) p.550 explica que, si una persona decide invertir, se está lanzando a una actividad empresarial que deberá gestionar siguiendo principios empresariales puros:

«Conozca lo que está haciendo, conozca su negocio». Aplicado a la inversión sería que no espere sacar buenos rendimientos, si no tiene amplios conocimientos sobre su apuesta.

«No permita que nadie dirija su empresa salvo (1) que pueda supervisar sus resultados con una diligencia adecuada y comprendiendo 
los datos o (2) salvo que tenga unos motivos inusualmente sólidos para depositar una confianza implícita en su integridad y su capacidad». Aplicado a la inversión sería que debería determinar las condiciones en las cuales va a permitir que otra persona decida lo que hace con su dinero.

«No hagas una operación, es decir, no fabrique o comercie con su artículo, salvo que después de realizar un cálculo fiable esté convencido de que tiene una buena probabilidad de conseguir un beneficio razonable. En particular, manténgase alejado de los proyectos en los que tiene poco que ganar y mucho que perder». Aplicado a la inversión sería que no realice operaciones en las que ponga en riesgo una parte injustificada de su capital.

«Tenga el coraje de reconocer su conocimiento y su experiencia. $\mathrm{Si}$ ha llegado a una conclusión a partir de los hechos y sabe que su juicio es sensato, póngalo en práctica, aunque los demás duden u opinen otra cosa». Lo mismo lo podemos aplicar a la inversión.

A lo largo de esta breve descripción se ha tendido a relacionar el Value Investing con la inversión en acciones de empresas. Es cierto que un gran número de value investors invierten exclusivamente en este tipo de activo financiero y, de hecho, hay algunos que rechazan cualquier otro tipo. ${ }^{31}$ Pero, tal y como explica Charlie Munger en la cita que hemos recogido al inicio de este apartado, podríamos definir simplemente el Value Investing como «comprar una cosa por menos de la que vale» ${ }^{32}$, por lo que podríamos realizar una inversión en valor sobre cualquier activo financiero. Por último, siguiendo la estructura del primer apartado relacionado con la Escuela Austriaca, vamos a incluir una tabla comparativa detallando las diferencias más importantes entre el método de inversión en valor y las teorías de inversión mainstream (que son las que se suelen enseñar en universidades y escuelas de negocios).

\footnotetext{
31 Ver García Paramés (2016).

32 Ver entrevista a Álvaro Guzmán de Lázaro (2017) sobre este tema.
} 


\section{CUADRO 2: VALUE INVESTING VS. MAINSTREAM INVESTING THEORY}

\begin{tabular}{|c|c|}
\hline INVERSIÓN EN VALOR & $\begin{array}{l}\text { TEORÍA MAINSTREAM DE } \\
\text { INVERSIÓN }{ }^{33}\end{array}$ \\
\hline -Concepto de la inversión- & -Concepto de la inversión- \\
\hline $\begin{array}{l}\text { Maximización del margen de } \\
\text { seguridad. }\end{array}$ & $\begin{array}{l}\text { Maximización del retorno esperado } \\
\text { asumiendo el mínimo riesgo posible. } \\
\text { Mean-variance framework. }\end{array}$ \\
\hline -Protagonista de la inversión- & -Protagonista de la inversión- \\
\hline Inversor empresario. & Homo economicus. \\
\hline $\begin{array}{l}\text { Conexión con la Teoría de la Acción } \\
\text { Humana y la Función Empresarial. }\end{array}$ & $\begin{array}{c}\text { Conexión con la Teoría de la Elección } \\
\text { Racional y la EMH }\end{array}$ \\
\hline -Concepto del retorno esperado- & -Concepto del retorno esperado- \\
\hline $\begin{array}{c}\text { Estimación de un rango de valoraciones } \\
\text { una vez se entiende el activo. Se } \\
\text { consideran aspectos cualitativos y } \\
\text { cuantitativos. }\end{array}$ & $\begin{array}{c}\text { Se calcula a través de la media de los } \\
\text { retornos previos. Sólo se tiene en cuenta } \\
\text { el pasado a la hora de predecir el } \\
\text { futuro. }{ }^{34}\end{array}$ \\
\hline -Concepto del riesgo- & -Concepto del riesgo- \\
\hline $\begin{array}{l}\text { Riesgo es la probabilidad de pérdida } \\
\text { permanente de nuestro capital que } \\
\text { afrontamos al invertir en un activo } \\
\text { financiero. Modelización empresarial. }{ }^{35}\end{array}$ & $\begin{array}{c}\text { El riesgo es la volatilidad del activo, } \\
\text { medida mediante la varianza o } \\
\text { desviación típica. Modelización } \\
\text { matemática. }\end{array}$ \\
\hline -Concepción de la información- & -Concepción de la información- \\
\hline $\begin{array}{l}\text { La información necesaria es dispersa y } \\
\text { desconocida. No mesurable. }\end{array}$ & $\begin{array}{l}\text { La información necesaria para el } \\
\text { modelo es conocida y mesurable. }\end{array}$ \\
\hline
\end{tabular}

${ }^{33}$ Me voy a referir al modelo CAPM de William Sharpe (1964) y John Lintner (1965) construido sobre el modelo de selección de carteras de Harry Markowitz (1959).

34 Existen otros métodos más complejos a la hora de predecir el retorno futuro de un activo. Para ello, usan modelos econométricos teniendo en cuenta una serie de variables y proyectándolas al futuro. Pero el problema de base sigue siendo el mismo: se basan simplemente en datos del pasado cuantitativos (ya que deben ser medibles) para predecir el futuro.

35 Así, Benjamin Graham (1949) dice: «the bona fide investor does not lose money merely because the market Price of his holdings declines; hence the fact that a decline may occur does not mean he is running the risk of a true los [...] This confusion may be avoided if we apply the concept of risk solely to a loss which either is realized through actual sale, or is caused by a significant deterioration in the company's position». 


\begin{tabular}{|c|c|}
\hline INVERSIÓN EN VALOR & $\begin{array}{l}\text { TEORÍA MAINSTREAM DE } \\
\text { INVERSIÓN }{ }^{33}\end{array}$ \\
\hline -Posibilidades de predicción específica- & -Posibilidades de predicción específica- \\
\hline $\begin{array}{l}\text { La predicción es limitada debido a la } \\
\text { incertidumbre inerradicable intrínseca } \\
\text { en el futuro. Humildad intelectual. }\end{array}$ & $\begin{array}{l}\text { La predicción es posible y existen } \\
\text { sofisticadas técnicas cuantitativas para } \\
\text { calcular la fiabilidad de esa predicción. }\end{array}$ \\
\hline -Importancia diversificación- & -Importancia diversificación- \\
\hline $\begin{array}{l}\text { Aunque algunos value investors la } \\
\text { consideran necesaria, muchos están en } \\
\text { contra de la diversificación excesiva. }{ }^{36} \\
\text { Normalmente diversifican por } \\
\text { regulación. }\end{array}$ & $\begin{array}{c}\text { Al ser el riesgo cuantificable por la } \\
\text { volatilidad, la diversificación se antoja } \\
\text { imprescindible para minimizar la covar } \\
\text { entre los activos que componen la } \\
\text { cartera. }\end{array}$ \\
\hline -Uso de las matemáticas- & -Uso de las matemáticas- \\
\hline Limitado y prudente. ${ }^{37}$ & $\begin{array}{l}\text { Lenguaje matemático y variables } \\
\text { cuantitativas. }\end{array}$ \\
\hline -Relación con el mundo empírico- & -Relación con el mundo empírico- \\
\hline El modelo se adapta al mundo real. & El mundo real se adapta al modelo. \\
\hline - Metodología - & - Metodología - \\
\hline Bottom up. & Top down. \\
\hline -Horizonte temporal- & -Horizonte temporal- \\
\hline $\begin{array}{l}\text { Depende de cada inversor, pero se suele } \\
\text { situar en el largo plazo. Más de } 7 \text { años. }\end{array}$ & $\begin{array}{l}\text { Es el mismo para todos los inversores y } \\
\text { suele ser de un año. }\end{array}$ \\
\hline -Posición relativa de diferentes autores- & -Posición relativa de diferentes autores- \\
\hline Graham, Buffett, Munger, Lynch. & Markowitz, Treynor, Sharpe, Lintner. \\
\hline
\end{tabular}

Me gustaría acabar este punto con una frase que recogida en la carta de 1996 de Berkshire Hathaway a sus accionistas en la que Buffett explica: «To invest successfully, you need not understand beta, efficient markets, modern portfolio theory, option pricing or

${ }^{36}$ Así, Warren Buffett dice: «Diversification is a protection against ignorance. It makes very little sense for those who know what they're doing»; y por su parte, su socio Charlie Munger dice: «A well-diversified portfolio needs just four stocks».

37 García Paramés: «No se requieren grandes habilidades matemáticas con una calculadora es suficiente. A veces hay que hacer una labor de psicólogo y entender por qué los demás no están invirtiendo en una compañía». 
emerging markets. You may, in fact, be better off knowing nothing of these».

\section{SIMILITUDES Y COMPATIBILIDADES}

Lo primero que llama la atención es que tanto el Value Investing como la Escuela Austriaca son corrientes en desuso en sus respectivos campos y que, además, sus fundadores se opusieron al paradigma anterior que imperaba en ese momento:

CUADRO 3: INVERSIÓN EN VALOR VS. ESCUELA AUSTRIACA (1)

\begin{tabular}{|c|c|}
\hline INVERSIÓN EN VALOR & ESCUELA AUSTRIACA \\
\hline -Corriente en desuso- & -Corriente en desuso- \\
\hline $\begin{array}{l}\text { A pesar de ser una metodología de } \\
\text { inversión que ha obtenido grandes } \\
\text { resultados a lo largo de prolongados } \\
\text { periodos de tiempo y que tiene en su } \\
\text { haber alguna de las mayores fortunas } \\
\text { del mundo de la inversión, y una larga } \\
\text { lista de inversores y gestores con } \\
\text { impresionantes track-records; es un } \\
\text { método que se encuentra en desuso } \\
\text { dentro del mundo financiero. }\end{array}$ & $\begin{array}{l}\text { A pesar de que se trata de una Escuela } \\
\text { de pensamiento que ha demostrado su } \\
\text { validez a la hora de explicar los } \\
\text { fenómenos económicos desde una base } \\
\text { humanista y multidisciplinar, se } \\
\text { mantiene como una corriente marginal } \\
\text { dentro de las ciencias económicas } \\
\text { (aunque sus aportaciones son, a } \\
\text { regañadientes, cada vez más } \\
\text { reconocidas por el mainstream). }\end{array}$ \\
\hline Alter ego: Economía neoclásica. & $\begin{array}{l}\text { Alter ego: Teoría mainstream de la } \\
\text { inversión. }\end{array}$ \\
\hline
\end{tabular}




\begin{tabular}{cc}
\hline INVERSIÓN EN VALOR & ESCUELA AUSTRIACA \\
\hline -Fundadores, cambio de paradigma- & -Fundadores, cambio de paradigma- \\
El origen de esta filosofía lo podemos & El origen de esta teoría económica lo \\
situar desde que Benjamin Graham y & podemos situar con la publicación en \\
David Dodd comenzaron a impartir & 1871 de la obra Principios de Economía \\
clase en la Columbia Business School en & $\begin{array}{c}\text { Política de Carl Menger. }{ }^{38} \text { En el contexto } \\
\text { de la publicación de esta obra, el }\end{array}$ \\
1928, publicando en 1934 su libro & pensamiento de la Escuela Clásica de \\
Security Analysis. Antes de Graham, a & Economía dominaba el estudio de la \\
principios del Siglo XX, sólo la renta fija & ciencia económica desde 1776 con la \\
se consideraba inversión, mientras que & publicación del libro La Riqueza de las \\
la compra de acciones se consideraba & Naciones de Adam Smith. Las ideas de \\
especulación. Esto se debía a que pocos & esta corriente, si bien defendían el libre \\
inversores utilizaban el análisis & mercado y la Ley de Say ${ }^{39}$, diferían de \\
fundamental para seleccionar sus & los pensamientos de Menger ya que \\
inversiones, decantándose por métodos & explicaban el mundo real a través de \\
sin ningún fundamento lógico o & conceptos objetivistas como la teoría del \\
financiero. & valor-trabajo, el equilibrio y otras \\
& muchas consideraciones erróneas e \\
& influidas por el calvinismo. ${ }^{40}$ \\
\hline
\end{tabular}

Además, podemos encontrar algunas curiosas coincidencias entre el origen del Value Investing y de la Escuela Austriaca. En la Nota 1 expongo las conclusiones a las que llega Chris Leithner (2005) tras la lectura de Benjamin Graham (1996) y Margit von Mises (1984).

38 Aunque exista un acuerdo generalizado de que en este momento nace la Escuela Austriaca de Economía, Huerta de Soto (2012) p.53 nos recuerda: «el principal mérito de este autor consiste en haber sabido recoger e impulsar una tradición del pensamiento de origen católico y europeo continental que se puede remontar hasta los albores del pensamiento filosófico en Grecia y, con mayor intensidad incluso, hasta la más rancia tradición del pensamiento jurídico, filosófico y político de la Roma clásica».

39 En resumen, podemos entender la La Ley de Say como que: "toda oferta genera su propia demanda". Keynes la malinterpretó, ya que Say quería explicar que la dirección de causalidad va desde la oferta hacia la demanda, es decir, la producción supone la generación simultánea de renta y capacidad de compra, lo cual aumenta la demanda.

${ }^{40}$ Menger no slo se opuso al paradigma anglosajón que dominaba en ese momento, sino que, en Alemania, cuando Gustav Schmoller llega a ser profesor titular en 1882 en la Universidad de Berlín y comenzaban a dominar las ideas de la Escuela Historicista Alemana, comenzó el Methodenstreit con su obra Investigations into de Method of the Social Sciences with Special Reference to Eocnomics (1883). 


\section{NOTA 1: CURIOSIDADES VALUE INVESTING Y ESCUELA AUSTRIACA}

\footnotetext{
«Ludwig von Mises, one of the twentieth century's most influential Austrian School economists, migrated to the United States in 1940. Sixteen years later, Benjamin Graham, the founder of modern security analysis, disbanded his investment company, returned its assets to shareholders and retired to southern California. During these years they both lived and worked in Manhattan, but trod a circuit without direct reference to the other. Mr Graham commuted between his home at 91st Street and Central Park West, his office at Graham-Newman Corp. and his securities analysis class at Columbia University; and Prof Mises trekked from his flat in West End Avenue to the National Association of Manufacturers, The New York Public Library, Foundation for Economic Education in Irvingtonon-Hudson and (beginning in 1949) his seminar at NYU's Graduate School of Business.

More generally, although Murray Rothbard (using a pseudonym) and Howard H.

Buffett (a Congressman and the father of Graham's most famous student, colleague and standard-bearer, Warren Buffett) commiserated during the 1950s about the bitter fruits of America's interventionist foreign and domestic policies, no publicly available information suggests that during these years Grahamite investors and Misesian economists crossed paths and exchanged ideas. Perhaps that is just as well. Had they met or corresponded, it is reasonable to suppose that a range of policy issues would have divided them. It is doubtful, for example, whether Graham's advocacy of a commodity-based reserve currency would have commanded Mises' assent; and it is still less likely that Graham's backing of the Employment Act of 1946 would have impressed Mises».
}

Aunque comprobamos que no existe una influencia directa clara en el origen, es curioso ver la cercanía y los puntos en común de ambas posturas. ${ }^{41} \mathrm{~A}$ continuación, vamos a estudiar la compatibilidad entre ambas teorías que se debe, básicamente, a una coincidencia en los principios básicos sobre los que se sustentan.

${ }^{41}$ De hecho, es también curioso que tanto la Acción Humana de Ludwig von Mises, como El Inversor Inteligente de Benjamin Graham fueron publicados en el mismo año, en 1949. 
CUADRO 4: INVERSIÓN EN VALOR VS. ESCUELA AUSTRIACA (2) $)^{42}$

\begin{tabular}{|c|c|}
\hline INVERSIÓN EN VALOR & ESCUELA AUSTRIACA \\
\hline $\begin{array}{l}\text {-El futuro es inerradicablemente } \\
\text { incierto- } 43\end{array}$ & $\begin{array}{l}\text {-El futuro es inerradicablemente } \\
\text { incierto- }\end{array}$ \\
\hline $\begin{array}{l}\text { En la inversión, si queremos realizar } \\
\text { una proyección sobre la posible } \\
\text { evolución de la empresa en el futuro, no } \\
\text { debemos considerar un escenario } \\
\text { optimista como punto de referencia } \\
\text { para tomar una decisión de inversión, } \\
\text { sino más bien un escenario muy } \\
\text { conservador como una forma de } \\
\text { protección. }\end{array}$ & $\begin{array}{c}\text { En el ámbito de la economía, hay } \\
\text { decisiones y libre albedrío. El futuro es } \\
\text { un porhacer y no un simple porvenir, } \\
\text { como suele recalcar el economista Jesús } \\
\text { Huerta de Soto. Para reducir la } \\
\text { incertidumbre al actuar existen algunos } \\
\text { mecanismos como las instituciones } \\
\text { sociales }{ }^{44} \text { o la propia función } \\
\text { empresarial. }\end{array}$ \\
\hline -Sentido común-46 & -Sentido común- \\
\hline $\begin{array}{l}\text { En la inversión, si se quiere adquirir un } \\
\text { activo, lo más razonable no es ignorar } \\
\text { su situación financiera (o la del } \\
\text { subyacente), ni su negocio, ni el precio } \\
\text { al que cotiza. Debemos realizar un } \\
\text { análisis pormenorizado y creativo. }\end{array}$ & $\begin{array}{l}\text { En economía, si se quiere desarrollar } \\
\text { una teoría económica, lo más razonable } \\
\text { sería tratar de que fueran nuestros } \\
\text { modelos los que se adapten a la realidad } \\
\text { y no que sea la realidad la que se adapte } \\
\text { a nuestros modelos. }\end{array}$ \\
\hline
\end{tabular}

42 Para realizar el siguiente cuadro comparativo me he basado en las ideas expuestas en el artículo de Diego E. Quijano Durán (2012) titulado "Vínculo entre la Escuela Austriaca de Economía y el método de inversión en valor".

43 Ambas posturas coindicen en que el futuro no está determinado y el mercado es visto como una consecución de interrelaciones entre personas en las que cada una persigue una serie de fines subjetivos. Esta realidad, donde se producen millones de decisiones al minuto, convierte al futuro en un concepto casado con la incertidumbre inerradicable.

44 Permiten reducir la incertidumbre ya que gracias a ellas podemos prever las conductas más probables de quienes nos rodean (los contratos se cumplen, la familia se protege, el lenguaje tiene unas reglas).

45 También permite reducir la incertidumbre ya que gracias a ella podemos prever qué personas tendrán éxito en lograr su objetivo según quién ejerza de mejor manera la función empresarial. Desde luego que no les podremos asignar probabilidades matemáticas, pero sí podemos ordenar a las personas o eventos según nuestras probabilidades (creo que el matrimonio de Pedro y María tiene más futuro que el de Pablo y Lucía).

46 Dice el aforismo popular, a veces atribuido al poeta español Antonio Machado, que el sentido común es el menos común de los sentidos. De ser así, ¿qué queremos decir con sentido común en este subapartado? En suma, que el conocimiento necesario para empezar a comprender y utilizar ambos marcos teóricos es muy elemental, sin 


\begin{tabular}{|c|c|}
\hline INVERSIÓN EN VALOR & ESCUELA AUSTRIACA \\
\hline -Supuestos realistas- 47 & -Supuestos realistas- \\
\hline $\begin{array}{l}\text { El Value Investing, no basará sus tesis } \\
\text { de inversión en análisis chartistas ni en } \\
\text { líneas de tendencia ficticias, que no } \\
\text { representan la situación actual del } \\
\text { activo. A este respecto, Jason Zweig } \\
\text { decía en una nota introductora del } \\
\text { Inversor Inteligente (1949): «A stock is not } \\
\text { just a ticker symbol or an electronic } \\
\text { blip; it is an ownership interest in an } \\
\text { actual business». }\end{array}$ & $\begin{array}{l}\text { En economía, cualquier ley económica } \\
\text { debe cumplir con el llamado } \\
\text { Individualismo Metodológico, es decir, } \\
\text { poder retrotraerse al comportamiento } \\
\text { del ser humano individual. A este } \\
\text { respecto, el concepto mainstream } \\
\text { sobreexplotado de equilibrio (en todas } \\
\text { sus vertientes) no es más que una } \\
\text { simplificación artificial que nunca } \\
\text { llegará a representar la realidad } \\
\text { dinámica del ser humano. }\end{array}$ \\
\hline -Prudencia al predecir-48 & -Prudencia al predecir- \\
\hline $\begin{array}{l}\text { En la Inversión en Valor, los analistas } \\
\text { no tratan de predecir con exactitud si el } \\
\text { precio que alcanzará un activo va a ser } \\
40,37 € \text { o } 41,23 € \text {. Su análisis se basa en } \\
\text { seleccionar activos subjetivamente } \\
\text { valiosos que crea que van a mantener } \\
\text { su posición competitiva. }\end{array}$ & $\begin{array}{l}\text { En economía, conscientes de la cantidad } \\
\text { de variables que influyen en cada } \\
\text { situación, no intentarán predecir si el } \\
\text { PIB va a crecer un 3,5\% o un 3,6\%. Sino } \\
\text { que, desde la modestia, se tratará de } \\
\text { resaltar los patrones de tendencia que } \\
\text { suelen ocurrir. }\end{array}$ \\
\hline -Microfundamentación-49 & -Microfundamentación- \\
\hline $\begin{array}{l}\text { En contra del agregacionismo. El Value } \\
\text { Investing suele enfocar su campo de } \\
\text { estudio en el análisis individualizado } \\
\text { del valor de un activo financiero. No } \\
\text { basan sus decisiones de inversión en el } \\
\text { comportamiento de agregados } \\
\text { macroeconómicos. }\end{array}$ & $\begin{array}{c}\text { En contra del agregacionismo. La } \\
\text { Escuela Austriaca concibe la ciencia } \\
\text { económica a partir de la acción humana } \\
\text { individual. Los análisis } \\
\text { macroeconómicos que utilizan un } \\
\text { excesivo agregacionismo, se alejan de } \\
\text { esa realidad que tratan de presentar los } \\
\text { austriacos. }\end{array}$ \\
\hline
\end{tabular}

requisitos de formación en sofisticadas técnicas matemáticas o conocimientos muy abstractos.

47 En relación con el punto anterior, el sentido común nos dirá que todo supuesto que utilicemos para elaborar una teoría económica o una metodología de inversión debe representar una realidad.

48 No se pueden realizar predicciones sobre eventos futuros con precisión. Esas múltiples decisiones de seres humanos individuales que conforman "la economía" son subjetivas, espontáneas y creativas, por lo que son difícilmente predecibles. Por ello, nos debemos limitar a identificar los patrones y características sobre la base de una serie de supuestos realistas.

49 Tienen una perspectiva realista del mundo por lo que no admiten trabajar sobre modelos irreales que utilizan variables agregadas. Por eso, como el foco lo ponen en el 


\begin{tabular}{|c|c|}
\hline INVERSIÓN EN VALOR & ESCUELA AUSTRIACA \\
\hline $\begin{array}{l}\text {-Prudencia en el uso de las } \\
\text { matemáticas- } 50\end{array}$ & $\begin{array}{l}\text {-Prudencia en el uso de las } \\
\text { matemáticas- }\end{array}$ \\
\hline $\begin{array}{l}\text { El Value Investing se despoja de todas } \\
\text { las pretensiones de las matemáticas } \\
\text { financieras más avanzadas, regresando } \\
\text { al uso de las relaciones contables } \\
\text { básicas y enfatizando la importancia de } \\
\text { su interpretación. Esto no quiere decir } \\
\text { que se trata de un rechazo absoluto a las } \\
\text { matemáticas, sino de enfatizar el hecho } \\
\text { de que no son las matemáticas y su } \\
\text { aparente precisión lo que dispensa el } \\
\text { conocimiento necesario para tomar } \\
\text { decisiones más o menos acertadas a la } \\
\text { hora de invertir. }\end{array}$ & $\begin{array}{l}\text { En la economía, deberíamos alejarnos } \\
\text { de modelos completamente } \\
\text { matematizados que se olvidan de la } \\
\text { representación del ser humano } \\
\text { individual. Las matemáticas son útiles } \\
\text { para economizar el lenguaje: escribir } \\
\text { 345+21 es más corto que trescientos } \\
\text { cuarenta y cinco más veintiuno; y facilitan } \\
\text { las operaciones a la hora de calcular. } \\
\text { Pero debemos ser prudentes al } \\
\text { utilizarlas y no caer en errores como el } \\
\text { de usar derivadas para calcular la } \\
\text { cantidad óptima que se debería } \\
\text { producir de un bien. }\end{array}$ \\
\hline -Beneficios acción empresarial-51 & -Beneficios acción empresarial- \\
\hline $\begin{array}{l}\text { Los value investors identifican errores } \\
\text { de valoración y procede a invertir en el } \\
\text { mercado de valores evitando empresas } \\
\text { sobrevaloradas e invirtiendo en } \\
\text { aquellas infravaloradas. Así, } \\
\text { contribuyen a ajustar desequilibrios y a } \\
\text { coordinar los distintos planes de los } \\
\text { participantes en el proceso de mercado. } \\
\text { Importancia empresarial. }\end{array}$ & $\begin{array}{l}\text { Los empresarios actúan identificando } \\
\text { oportunidades en el mercado que no } \\
\text { están satisfechas. Cubrir esas demandas } \\
\text { implica utilizar los recursos con mayor } \\
\text { eficiencia (se eliminan más desajustes } \\
\text { con menos recursos), lo cual abre la } \\
\text { oportunidad de utilizar los recursos } \\
\text { liberados para satisfacer nuevos } \\
\text { desajustes. Coordinación dinámica. }\end{array}$ \\
\hline
\end{tabular}

empresario, en las personas de carne y hueso, podemos decir que ambos enfoques están microfundamentados.

${ }^{50}$ Las matemáticas se enfrentan a dos principales problemas. El primero es, como dice el economista Juan Carlos Cachanosky, que "no todo lo que se dice en palabras es traducible a matemática, pero toda formulación matemática es traducible a palabras". El segundo, no son una herramienta útil para conocer más sobre la realidad, se pueden utilizar para facilitar la representación de una situación, pero no para sacar conclusiones sobre fenómenos económicos.

${ }^{51}$ Los value investor son empresarios kirznerianos (se detallará en el segundo artículo de esta colección) que ejercen un papel coordinador en el mercado a través de la función empresarial. Ambos son conscientes que mientras inversores y empresarios mejor realicen sus actividades, mayor será la eficiencia en el mercado. 


\begin{tabular}{|c|c|}
\hline INVERSIÓN EN VALOR & ESCUELA AUSTRIACA \\
\hline -Optimismo a largo plazo- 52 & -Optimismo a largo plazo- \\
\hline $\begin{array}{l}\text { En el Value Investing impera una visión } \\
\text { positiva acerca de la evolución del } \\
\text { mercado en general y de sus inversiones } \\
\text { en particular. Por esta razón insisten en } \\
\text { aplicar un horizonte temporal } \\
\text { largoplacista en sus inversiones. }\end{array}$ & $\begin{array}{c}\text { En la economía, sabemos que el proceso } \\
\text { empresarial de coordinación social } \\
\text { jamás se detiene ni se agota. Siempre } \\
\text { aparecerán nuevos desajustes que } \\
\text { suponen nuevas oportunidades de } \\
\text { ganancia empresarial. Proceso } \\
\text { dinámico evolutivo. }\end{array}$ \\
\hline -Concepto de riesgo- 53 & Concepto de riesgo \\
\hline $\begin{array}{l}\text { En la inversión, la mayoría de riesgos a } \\
\text { los que se enfrenta el inversor están } \\
\text { asociados con lo que no se podía } \\
\text { predecir. Los value investors son } \\
\text { conscientes de esto y dan más } \\
\text { importancia a conceptos como los black } \\
\text { swans }{ }^{54} \text { que a medidas estandarizadas } \\
\text { de riesgos matematizables como el VaR. }\end{array}$ & $\begin{array}{c}\text { En economía, la mayoría de los riesgos a } \\
\text { los que se enfrenta un empresario no } \\
\text { son asegurables, ni mesurables. Cada } \\
\text { evento tiene carácter abierto e ilimitado. } \\
\text { Por lo tanto, el riesgo hace referencia a } \\
\text { la incertidumbre inerradicable y no } \\
\text { matematizable. }\end{array}$ \\
\hline
\end{tabular}

En este apartado vamos a hacer un análisis de las discrepancias entre ambos enfoques. Existe un debate abierto en la Academia concentrado en este punto ya que todavía no hay una respuesta clara sobre su grado de compatibilidad. En la siguiente tabla introduciremos los puntos de máxima confrontación y trataremos de dar una explicación lógica en los pies de página.

52 Ambos consideran que el mercado se encuentra en un estado de desequilibrio y que, a través de la función empresarial, tiende a coordinarse. Como conocen que la causa última de esa coordinación es la acción humana y que es inherente a todo ser humano, son optimistas sobre el futuro.

53 El concepto de riesgo que típicamente encontramos en ciencias sociales, hace referencia al estudio de la probabilidad de que ocurra un evento que ya conocemos que puede suceder. Sin embargo, tanto la Escuela Austriaca como el Value Investing insisten en que el riesgo reside en lo no conocido.

54 Ver el libro de Nassim Nicholas Taleb (2007) titulado The Black Swan. Existe una traducción al español por la editorial Planeta (2012) con el título El Cisne Negro. 
CUADRO 5: INVERSIÓN EN VALOR VS. ESCUELA AUSTRIACA (3)

\begin{tabular}{|c|c|}
\hline \multicolumn{2}{|c|}{ RACIONALIDAD vs. IRRACIONALIDAD ${ }^{55}$} \\
\hline -visión escuela austriaca- & -visión value investing- \\
\hline $\begin{array}{l}\text { Para los economistas austriacos } \\
\text { cualquier acción humana es siempre } \\
\text { racional. }\end{array}$ & $\begin{array}{l}\text { Los value investors inciden en que las } \\
\text { diferencias entre precio y valor, se } \\
\text { deben al comportamiento irracional de } \\
\text { los diferentes agentes financieros. }\end{array}$ \\
\hline $\begin{array}{c}\text { Mises (1949): «Human action is } \\
\text { necessarily always rational». }\end{array}$ & $\begin{array}{l}\text { Graham y Dodd (1934): «Obviously the } \\
\text { stock market is quite irrational in thus } \\
\text { varying its valuation of a company } \\
\text { proportionately with the temporary } \\
\text { changes in its reported profits». }\end{array}$ \\
\hline \multicolumn{2}{|c|}{ VALORACIÓN vs. ESTIMACIÓN 56} \\
\hline -visión escuela austriaca- & -visión value investing- \\
\hline $\begin{array}{c}\text { Valorar consiste en ordenar los bienes } \\
\text { en una escala subjetivamente, y estimar } \\
\text { consiste en anticipar algún evento } \\
\text { futuro. }\end{array}$ & $\begin{array}{l}\text { Los value investors, sin embargo, no } \\
\text { inciden en tal diferencia y en ocasiones } \\
\text { utilizan los términos como sinónimos. }\end{array}$ \\
\hline $\begin{array}{l}\text { Mises (1949): «Appraisement must be } \\
\text { clearly distinguished from valuation. } \\
\text { Appraisement in no way depends upon } \\
\text { the subjective valuation of the man who } \\
\text { appraises». }\end{array}$ & $\begin{array}{l}\text { Graham (1949): «Let us return for a } \\
\text { moment to the idea of valuation or } \\
\text { appraisal of a common stock...». }\end{array}$ \\
\hline
\end{tabular}

${ }^{55}$ Los economistas de la Escuela Austriaca son académicos y los value investors son inversores prácticos. Los primeros usarán un lenguaje más preciso y depurado que los segundos. Hablar de irracionalidad en los mercados es un claro ejemplo de ello, ya que es impreciso y no profundiza sobre el significado de lo que está explicando. Los mercados no tienen personalidad propia y si suben o bajan de manera "irracional", lo que estará sucediendo es que millones de inversores individuales habrán tomado la decisión de invertir "racionalmente" (deliberadamente). Si finalmente esa inversión resulta en un error, habrán cometido un error empresarial, pero nadie puede negar que el inversor en el momento de invertir, tomó la decisión que pensaba que era la correcta con la información que tenía a su disposición.

56 Otro ejemplo de lo que comentábamos en el punto anterior. El value investor no se detiene a reflexionar sobre la diferencia entre los dos términos porque simplemente se dedica a llevar a la práctica una metodología para invertir cargada de conocimiento práctico pero que no es muy rigurosa con la precisión de los aspectos teóricos. Por lo tanto, no creo que haya una incompatibilidad insalvable entre ambos enfoques, simplemente un error de precisión. 


\begin{tabular}{|c|c|}
\hline \multicolumn{2}{|c|}{ VALOR SUBJETIVO vs. VALOR INTRÍNSECO 57} \\
\hline - visión escuela austriaca - & - visión value investing - \\
\hline $\begin{array}{l}\text { La acción humana depende de fines y } \\
\text { medios que la persona valora } \\
\text { subjetivamente. Cada bien tendrá un } \\
\text { valor diferente entre las diferentes } \\
\text { personas (incluso para la misma } \\
\text { persona en diferentes momentos). }\end{array}$ & $\begin{array}{l}\text { Los value investors defienden la } \\
\text { existencia de un valor intrínseco que es, } \\
\text { por definición, objetivo. Es más, el } \\
\text { margen de seguridad es la diferencia } \\
\text { entre el precio de mercado y el valor } \\
\text { intrínseco del activo. }\end{array}$ \\
\hline $\begin{array}{l}\text { Mises (1949): «Value is not intrinsic; it is } \\
\text { not in things. It is within us; it is the } \\
\text { way in which man reacts to the } \\
\text { conditions of his environment. Neither } \\
\text { is value in words and doctrines, it is } \\
\text { reflected in human conduct. It is not } \\
\text { what a man or groups of men say about } \\
\text { value that counts, but how they act». }\end{array}$ & $\begin{array}{l}\text { Graham y Dodd (1934): «In all of these } \\
\text { instances he appears to be concerned } \\
\text { with the intrinsic value of the security } \\
\text { and more particularly with the } \\
\text { discovery of discrepancies between the } \\
\text { intrinsic value and the market price». }\end{array}$ \\
\hline
\end{tabular}

INVERSIÓN vs. ESPECULACIÓN ${ }^{58}$

57 Este es el foco principal sobre el que se sustentan las críticas entre la compatibilidad de ambos enfoques. ¿Cómo van a ser compatibles dos enfoques que parece que están en las antípodas de lo que entienden por valor? Bien, pues creo que es el punto más sencillo de defender. Los propios value investors alertan de que el concepto de valor intrínseco no debe tomarse como un valor objetivo alcanzable. Así, leemos en Graham y Dodd (1934): "intrinsic value is an elusive concept", Buffett admite que el concepto de valor intrínseco es "fuzzy" (vago), pero creo que la persona que mejor lo define en García Paramés (2011): "nuestro trabajo consiste en encontrar discrepancias entre los precios actuales, o para ser más exactos, los precios que acaban de formarse en el pasado reciente, $y$ las valoraciones que los agentes van a realizar de los activos en el futuro y que van a determinar los precios futuros. Lo que analizamos, en suma, son discrepancias entre valoraciones subjetivas actuales y valoraciones subjetivas futuras". Por lo tanto, vemos que, de nuevo, es un problema de rigor en los términos utilizados, pero no de concepto.

58 Creo que la discrepancia se debe a que están utilizando la palabra "especulación" para referirse a dos conceptos diferentes. Los economistas austriacos se refieren a "especulador" como el concepto que proviene del latín specula (torre elevada a la que subían los vigías para tratar de ver con mucha antelación los movimientos del enemigo), por lo tanto, sería la persona que intenta prever los comportamientos de los consumidores y actuar para satisfacer esas demandas futuras. Los value investors se refieren a un concepto peyorativo (con el que posiblemente estemos más familiarizados) que representa una manera de obtener beneficios arriesgando todo el capital inicial con el fin de aprovecharse de las fluctuaciones en los precios. De nuevo, problema semántico. 


\begin{tabular}{|c|c|}
\hline -visión escuela austriaca- & -visión value investing- \\
\hline $\begin{array}{c}\text { El empresario es un especulador } \\
\text { perspicaz que trata de anticiparse a los } \\
\text { deseos que tendrán los consumidores } \\
\text { en el futuro. }\end{array}$ & $\begin{array}{l}\text { Los value investors, sin embargo, } \\
\text { marcan una clara diferencia entre los } \\
\text { que es invertir y lo que especular. }\end{array}$ \\
\hline $\begin{array}{l}\text { Mises (1962): «Every action is a } \\
\text { speculation, i.e., guided by a definite } \\
\text { opinion concerning the uncertain } \\
\text { conditions of the future». }\end{array}$ & $\begin{array}{l}\text { Graham (1949): «The distinction } \\
\text { between investment and speculation in } \\
\text { common stocks has always been a } \\
\text { useful one and its disappearance is a } \\
\text { cause for concern». }\end{array}$ \\
\hline
\end{tabular}

\section{REFERENCIAS BIBLIOGRÁFICAS}

Buffett, W. E. (1984): The Superinvestors of Graham-and-Doddsville, Columbia Business School magazine.

— (1987, 1989, 1996): Berkshire Hathaway Inc.'s Shareholder Letters.

- (1998): «Warren Buffett speaks with Florida University». Youtube.

- (2011): «Warren Buffett Remarks on European Debt Crisis, the "Buffett Rule" and the American Worker». Entrevista en Business Wire.

Ceolin, A. M. (2019): «Contrastando el papel de la serendipidad en las teorías austriaca y neoclásica», en el Mises Journal de Brasil, Vol. 8 (2020): Yearly Continuous Publication, publicado el 29 de noviembre de 2019. https://misesjournal.org.br/misesjournal/ article/view/1251

Cunningham, L. \& Eide, T. \& Hargreaves, P. (2016): Invirtiendo en calidad: Claves para invertir a largo plazo en las mejores empresas, Barcelona, Deusto [2018].

Dorsey, P. (2008): El pequeño libro que genera riqueza: La fórmula definitiva para encontrar inversiones excelentes, Deusto [2016].

García Paramés, F. (2016): Invirtiendo a largo plazo: Mi experiencia como inversor, Barcelona, Deusto.

Graham, B. \& Dodd, D. L. (1934): Security analysis: Principles and technique. New York, McGraw-Hill, [1951].

Graham, B. (1949): El inversor inteligente, Barcelona, Deusto, Grupo Planeta, [17 a ed. 2017]. 
- (1996). Benjamin Graham: The Memoirs of the Dean of Wall Street, McGraw-Hill.

Grant, J. (1996): Interview: «The Trouble with Prosperity», Austrian Economics Newsletter, Winter 1996, Vol. 16, N. 4.

Guzmán de Zalaro, A. (2017): «¿Qué es el ‘value investing'?», Azvalor you. Youtube.

Huerta de Soto, J. (2009): "La esencia de la Escuela Austriaca." Discurso pronunciado al recibir el doctorado honoris causa en Ciencias Sociales, otorgado por la Universidad Francisco Marroquín. Guatemala, 2 de mayo del 2009.

- (1998): Dinero, crédito bancario y ciclos económicos, Unión Editorial, Madrid, [7 $7^{\mathrm{a}}$ ed. 2020].

- (2000): La Escuela Austriaca: mercado y creatividad empresarial, Madrid, Editorial Síntesis, [2 ${ }^{\mathrm{a}}$ ed. 2012].

- (1992): Socialismo, cálculo económico y función empresarial, Madrid, Unión Editorial [6a ed. 2020].

Keynes, J. M. (1991): The General Theory of Employment, Interest and Money, Palgrave Macmillan.

Klarman, S. A. (1991): Margin of Safety: Risk-Averse Value Investing Strategies for the Thoughtful Investor, HarperCollins.

Leithner, C. (2015): «Entrepreneurship and Intelligent Investing», Le Québécois Libre.

- (2005): «Ludwig von Mises, Meet Benjamin Graham: Value Investing from an Austrian Point of View», en Mises.org, preparado para la conferencia «Austrian Economics and Financial Markets», Las Vegas, 18-19 de febrero de 2005.

Lintner, J. (1965): «The Valuation of Risk Asset and the Selection of Risky Investments in Stock Portfolios and Capital Budgets», The Review of Economics and Statistics: The MIT Press.

Lynch, $\mathrm{P}$.

- (1989): Un paso por delante de Wall Street: cómo utilizar lo que ya sabes para ganar dinero en bolsa, Barcelona, Ediciones Deusto [2015].

Markowitz, H. M. (1959): Portfolio Selection, Efficient Diversification of Investments, New York: John Wiley and Sons.

Martínez Meseguer, C. (2006): La teoría evolutiva de las instituciones: la perspectiva austriaca, Madrid, Unión Editorial [2ª ed. 2016]. Mendoza, E. (1991): Sin Noticias de Gurb, Madrid, Seix Barral [2001]. 
Menger, C. (1883): Investigations into the Method of the Social Sciences. New York: New York University Press [1985].

- (1871): Principles of Economics. Alabama: Ludwig von Mises Institute [2007].

- (1892): The Origins of Money. Alabama: Ludwig von Mises Institute [2009].

von Mises, L. (1949): La Acción Humana., Madrid, Unión Editorial [12a Edición 2018].

- (1962): The Ultimate Foundation of Economic Science: An Essay on Method, Liberty Fund [2006].

von Mises, M. (1984): My Years With Ludwig von Mises. Libertarian Press.

Munger, C. T. (1883): Poor Charlie's Almanack, Missouri: The Donning Company Publishers [Expanded Third Edition 1985].

Quijano, D. E. (2011): «La inversión en valor y la economía austriaca», La Ilustración Liberal, N. 50 (invierno 2011): 37-42.

- (2012): «Vínculos entre la escuela austriaca de economía y el método de inversión en valor», en Procesos de Mercado: Revista Europea de Economía Política, Vol. 9, No. 1, 2012, págs. 255-304.

Quintana, E. (2016): «Value Investing: Una fundamentación teórica desde el punto de vista de la Escuela Austriaca de Economía», Trabajo Fin de Máster en Economía de la Escuela Austriaca URJC.

Rallo, J. R. (2010): «Escuela Austriaca y value investing: una hoja de ruta», Instituto Juan de Mariana.

- (2010): «Los Fundamentos Económicos del Value Investing», Juan Ramón Rallo website.

- (2019): La Ruta Value. Value School.

Sharpe, W. F. (1964): «Capital Asset Prices: A Theory Of Market Equilibrium Under Conditions Of Risk», The Journal of Finance, Volume 19, Issue 3.

Siegel, J. J. (1994): Stocks for the Long Run, 5 ed., McGraw-Hill Education [2014].

Smith, A. (1776): La riqueza de las naciones, Grupo Anaya Publicaciones Generales [2011].

Taleb, N. N. (2007): El cisne negro: el impacto de lo altamente improbable, Barcelona: Booket [2012]. 
Trucmel, A. (2019): «Malinterpretando el Value Investing». Value School.

Vanhaverbeke, F. (2014): Excess Returns: A Comparative Study of the Methods of the World's Greatest Investors, Harriman House. 